\title{
SPACES OF GENERALIZED ANALYTIC FUNCTIONS
}

\author{
By \\ D. E. MYERS (Tucson)
}

This author in some previous papers [5], [6], [7] has investigated certain properties of complex functions analytic in a strip in the complex plane. The strip was considered because it is the natural domain for functions that are Laplace transforms. MACKey [3], Ellot [2], ARENS and Singer [1] among others have given definitions of analytic functions defined on some subset of $\bar{G} \times \hat{G}$, the generalized character group of a locally compact Abelian Group. Mackey and Elliot used derivatives with respect to semigroups, Arens and Singer the duality contained in a Poisson representation. None of the above papers contain any elaboration of the properties of the class of analytic functions like the Cauchy-Riemann conditions or preservation of analyticity under uniform convergence. One result of this paper is the existence of necessary and sufficient conditions for analyticity which are analogous to the Cauchy-Riemann equations. In this paper I will also obtain those extensions which allow us to construct a Hilbert space of such functions.

Notation. Let $G$ be a locally compact group (not necessarily Abelian) and $\hat{G}$ its character group. That is, $\hat{G}$ consists of those complex-valued functions, continuous on $G$ with modulus identically 1 and with a multiplicative property. $\hat{G}$ is also a locally compact group and the topology is that of uniform convergence on compact subsets of $G . \bar{G}$ denotes the set of real, continuous linear functionals on $G$. A subset $K$, of $\bar{G}$ is said to be large convex if it is convex, contains the zero element and the closed linear span is $\bar{G} . \bar{G} \times \bar{G}$ becomes a complex vector space by defining $(u+i v)\left(x_{1}, x_{2}\right)=\left(u x_{1}-v x_{2}, v x_{1}+u x_{2}\right)$ for $u+i v$ complex and $\left(x_{1}, x_{2}\right) \in \bar{G} \times \bar{G}$. Finally for each $x \in \bar{G}$, define a one-parameter subgroup of $\hat{G}$ by $x[u]=\exp ($ iux $)$, $u$ real.

DEFINITION (MACKEY [4]). Let $K$ be a large convex subset of $\bar{G}$ and $x_{0}$ an interior point of $K$ (i.e. there exists $r>1$ such that $r x \in K$ ). Let $F(x, y)$ be a complex-valued function defined on $K \times \hat{G}$. It is said to be analytic at $\left(x_{0}, y_{0}\right)$ if

$$
\operatorname{Lim}_{u \rightarrow 0} \frac{F\left(x_{0}+u x_{1}, y_{0} x_{2}[u]\right)-F\left(x_{0}, y_{0}\right)}{u}=F_{x_{1}, x_{2}}\left(x_{0}, y_{0}\right)
$$

exists for every $\left(x_{1}, x_{2}\right) \in \bar{G}$.

(ii) The above limit is a complex-homogeneous function of $\left(x_{1}, x_{2}\right)$. As will be shown the complex-homogeneity condition is analogous to requiring that the partial derivatives satisfy Cauchy-Riemann like conditions. 
LEMMA 1.1. If $F(x, y)$ is analytic at $(x, y) \in \bar{G} \times \hat{G}$ and $U(x, y), V(x, y)$ denote real valued functions such that $F(x, y)=U(x, y)+i V(x, y)$ then $U_{\left(x_{1}, x_{2}\right)}(x, y)$, $V_{\left(x_{1}, x_{2}\right)}(x, y)$ exist as complex-homogeneous functions of $\left(x_{1}, x_{2}\right)$ and

$$
U_{\left(x_{1}, 0\right)}=V_{\left(0, x_{1}\right)}, \quad U_{\left(0, x_{1}\right)}=-V_{\left(x_{1}, 0\right)}
$$

for all $x_{1} \in \bar{G}$.

Proof. Since $0 \in \bar{G}, F_{\left(x_{1}, 0\right)}$ exists and $F_{\left(x_{1}, 0\right)}=U_{\left(x_{1}, 0\right)}+i V_{\left(x_{1}, 0\right)}$. Since it follows easily from the definition that the differentiation is linear. Further $i\left(x_{1}, 0\right)=$ $=\left(0, x_{1}\right)$ so that

$$
i F_{\left(x_{1}, 0\right)}=i U_{\left(x_{1}, 0\right)}-V_{\left(x_{1}, 0\right)}=F_{\left(0, x_{1}\right)}=U_{\left(0, x_{1}\right)}+i V_{\left(0, x_{1}\right)}
$$

and hence

$$
U_{\left(x_{1}, 0\right)}=-V_{\left(x_{1}, 0\right)}, \quad V_{\left(0, x_{1}\right)}=U_{\left(x_{1}, 0\right)} .
$$

LEMMA 1.2. Let $(x, y) \in \bar{G} \times \hat{G}, N$ a convex neighbourhood of $(x, y)$ such that at each point $\left(x^{\prime}, y^{\prime}\right)$ in $N$ and for all $x_{1} \in \bar{G}, U_{\left(x_{1}, 0\right)}\left(x^{\prime}, y^{\prime}\right)$ exists. Then there is a $\delta>0$ such that for $0<\alpha<\delta$, there is a $0<\beta(\alpha)<\alpha$ where

$$
U\left(x+\alpha x_{1}, y\right)-U(x, y)=\alpha U_{\left(x_{1}, 0\right)}\left(x+\beta x_{1}, y\right) .
$$

Proof. Let $g(w)=U\left(x+w x_{1}, y\right)$, then there exists $\delta>0$ such that $g^{\prime}(w)$ exists for $0 \leqq w \leqq \delta$ since

$$
\begin{gathered}
g^{\prime}(w)=\operatorname{Lim}_{\Delta w \rightarrow 0} \frac{U\left(x+(w+\Delta w) x_{1}, y\right)-U\left(x+w x_{1}, y\right)}{\Delta w}= \\
=\operatorname{Lim}_{\Delta w \rightarrow 0} \frac{U\left(x+w x_{1}+\Delta w x_{1}, y\right)-U\left(x+w x_{1}, y\right)}{\Delta w}=U_{\left(x_{1}, 0\right)}\left(x+w x_{1}, y\right)
\end{gathered}
$$

which by hypothesis exists for all $\left(x+w x_{1}, y\right) \in N . \delta$ may be taken to be any positive number such that $\left(x+\delta x_{1}, y\right) \in N$, by convexity $\left(x+\alpha x_{1}, y\right) \in N$ for $0 \leqq \alpha \leqq \delta$. Applying the Mean-Value Theorem to $g$ we have

for some $0<\beta<\alpha$, or

$$
g(\alpha)-g(0)=\alpha g^{\prime}(\beta)
$$

$$
U\left(x+\alpha x_{1}, y\right)-U(x, y)=\alpha U_{\left(x_{1}, 0\right)}\left(x+\beta x_{1}, y\right) .
$$
then

COROLLARY. If further $U_{\left(x_{1}, 0\right)}$ is a complex homogeneous function of $\left(x_{1}, 0\right)$

$$
U(x, y)-U\left(x+\alpha x_{1}, y\right)=i \alpha U_{\left(0, x_{1}\right)}\left(x+\beta x_{1}, y\right) .
$$

LEMMA 1.3. Let $(x, y)$ be in $\bar{G} \times \hat{G}$ and $N$ a convex neighbourhood of $(x, y)$ such that for all $\left(x^{\prime}, y^{\prime}\right)$ in $N$ and all $x_{1}$ in $\bar{G}, U_{\left(0, x_{1}\right)}\left(x^{\prime}, y^{\prime}\right)$ exists. Then there exists $\delta>0$ such that for all $0<\alpha<\delta$, there exists $0<\beta(\alpha)<\alpha$ such that

$$
U\left(x, y x_{1}[\alpha]\right)-U(x, y)=\alpha U_{\left(0, x_{1}\right)}\left(x, y x_{1}[\beta]\right) .
$$


Proof. Let $h(w)=U\left(x, y x_{1}[w]\right)$ and the proof proceeds as for Lemma 1 if we note that

$$
U\left(x, y x_{1}[w+\Delta w]\right)=U\left(x, y x_{1}[w] x_{1}[\Delta w]\right) .
$$

COROLLARY. If further $U_{\left(0, x_{1}\right)}$ is a complex homogeneous function of $\left(0, x_{1}\right)$ then

$$
U(x, y)-U\left(x, y x_{1}[\alpha]\right)=-i a U_{\left(x_{1}, 0\right)}\left(x, y x_{1}[\beta]\right) .
$$

COROLLARY. If further $U_{\left(0, x_{1}\right)}$ is a complex homogeneous function of $\left(0, x_{1}\right)$, (and hence $U_{\left(x_{1}, 0\right)}$ is of $\left.\left(x_{1}, 0\right)\right)$ then

$$
U\left(x, y x_{1}[\alpha]\right)-U\left(x+\alpha x_{1}, y\right)=i x\left[U_{\left(x_{1} 0\right)}\left(x, y x_{1}\left[\beta^{\prime}\right]\right)-U_{\left(0, x_{1}\right)}\left(x+\beta^{\prime \prime} x_{1}, y\right)\right]
$$

for some $0<\beta^{\prime}<\alpha, 0<\beta^{\prime \prime}<\alpha$ or

$$
U\left(x+\alpha x_{1}, y\right)-U\left(x, y x_{1}[\alpha]\right)=\alpha\left[U_{\left(x_{1}, 0\right)}\left(x+\beta^{\prime \prime} x_{1}, y\right)-U_{\left(0, x_{1}\right)}\left(x, y x_{1}\left[\beta^{\prime}\right]\right)\right] .
$$

LEMMA 1.4. Let $F(x, y)=U(x, y)+i V(x, y)$ and suppose that for all $x_{1} \in \bar{G}$, $U\left(x^{\prime}, y^{\prime}\right), V\left(x^{\prime}, y^{\prime}\right), U_{\left(x_{1}, 0\right)}\left(x^{\prime}, y^{\prime}\right), V_{\left(x_{1}, 0\right)}\left(x^{\prime}, y^{\prime}\right)$ exist and are continuous in a convex neighbourhood of $(x, y)$. Then if $U_{\left(0, x_{1}\right)}(x, y)=-V_{\left(x_{1}, 0\right)}(x, y), U_{\left(x_{1}, 0\right)}(x, y)=V_{\left(0, x_{1}\right)}$ $(x, y)$ for all $x_{1}$ in $\widetilde{G}, F(x, y)$ is analytic at $(x, y)$.

Proof. It is clearly sufficient to show that

$$
(\alpha+i \beta) \operatorname{Lim}_{u \rightarrow 0} \frac{F\left(x+u x_{1}, y x_{2}[u]\right)-F(x, y)}{u}
$$

and

$$
\operatorname{Lim}_{u \rightarrow 0} \frac{F\left(x+\alpha u x_{1}-\beta u x_{2}, y x_{2}[\alpha u] x_{1}[\beta u]\right)-F(x, y)}{u}
$$

exist and are equal for all $\alpha+i \beta \in C$ and $\left(x_{1}, x_{2}\right) \in \bar{G} \times \bar{G}$. Writing $F(x, y)=U(x, y)+$ $+i V(x, y)$ we may consider $U$ and $V$ separately.

(1) Consider

$$
\begin{gathered}
\frac{\left.U\left(x+\alpha u x_{1}-\beta u x_{2}, y x_{2}[\alpha u] x_{1}[\beta u]\right)\right)-U(x, y)}{u}= \\
=\alpha\left[\frac{U\left(x+\alpha u x_{1}-\beta u x_{2}, y x_{2}[\alpha u] x_{1}[\beta u]\right)-U\left(x-\beta u x_{2}, y x_{2}[\alpha u] x_{1}[\beta u]\right)}{\alpha u}\right]+ \\
+\alpha \frac{U\left(x-\beta u x_{2}, y x_{2}[\alpha u] x_{1}[\beta u]\right)-U\left(x-\beta u x_{2}, y x_{1}[\beta u]\right)}{\alpha u}+ \\
+\beta\left[\frac{U\left(x-\beta u x_{2}, y x_{1}[\beta u]\right)-U\left(x-\beta u x_{2}, y\right)}{\alpha u}\right]-\beta\left[\frac{U\left(x-\beta u x_{2}, y\right)-U(x, y)}{-\beta u}\right] .
\end{gathered}
$$


If we apply Lemma 2 and Lemma 3 then we obtain

$$
\begin{gathered}
\frac{U\left(x+\alpha u x_{1}-\beta x_{2}, y x_{2}[\alpha u] x_{1}[\beta u]\right)-U(x, y)}{u}= \\
=\alpha U_{\left(x_{1}, 0\right)}\left(x-\beta u x_{2}+\delta x_{1}, y x_{2}[\alpha u] x_{1}[\beta u]\right)+\alpha U_{\left(0, x_{2}\right)}\left(x-\beta u x_{2}, y x_{2}[\beta u] x_{2}\left(\delta^{\prime}\right]\right)+ \\
+\beta U_{\left(0, x_{1}\right)}\left(x-\beta u x_{2}, y x_{1}\left[\delta^{\prime \prime}\right]\right)-\beta U_{\left(x_{2} 0\right)}\left(x-\delta^{\prime \prime \prime} x_{2}, y\right)
\end{gathered}
$$

where $0<\delta, \delta^{\prime}<\alpha u, 0<\delta^{\prime \prime}, \delta^{\prime \prime \prime}<\beta u$. Using the continuity of the partials however, as $u \rightarrow 0$ we obtain

$$
\alpha U_{\left(x_{1}, 0\right)}(x, y)+\alpha U_{\left(0, x_{2}\right)}(x, y)+\beta U_{\left(0, x_{1}\right)}(x, y)-\beta U_{\left(x_{2}, 0\right)}(x, y) .
$$

(2) Proceeding in a similar fashion for

$$
\frac{V\left(x+\alpha u x_{1}-\beta u x_{2}, y x_{2}[\alpha u] x_{1}[\beta u]\right)-V(x, y)}{u}
$$

we obtain, as $u \rightarrow 0$

$$
\alpha V_{\left(x_{1}, 0\right)}(x, y)+\alpha V_{\left(0, x_{2}\right)}(x, y)+\beta V_{\left(0, x_{1}\right)}(x, y)-\beta V_{\left(x_{2}, 0\right)}(x, y) .
$$

Now utilizing the "Cauchy-Riemann" conditions we obtain

$$
\begin{gathered}
\operatorname{Lim}_{u \rightarrow 0} \frac{\left.\left.F\left(x+\alpha u x_{1}-\beta u x_{2}, y x_{2}[\alpha u] x_{1}[\beta u]\right)-F\right) x, y\right)}{u}= \\
=\alpha U_{\left(x_{1}, 0\right)}(x, y)+\alpha U_{\left(0, x_{2}\right)}(x, y)-\beta V_{\left(x_{1}, 0\right)}(x, y)-\beta V_{\left(0, x_{2}\right)}+ \\
+\alpha V_{\left(x_{1}, 0\right)}(x, y)+\alpha V_{\left(0, x_{2}\right)}(x, y)+i \beta U_{\left(x_{1}, 0\right)}(x, y)+i \beta U_{\left(0, x_{2}\right)}(x, y)= \\
=(\alpha+i \beta)\left[U_{\left(x_{1}, 0\right)}(x, y)+U_{\left(0, x_{2}\right)}(x, y)\right]+(\alpha+i \beta)\left[i V_{\left(x_{1}, 0\right)}(x, y)+i V_{\left(0, x_{2}\right)}\right]= \\
=(\alpha+i \beta)\left[U_{\left(x_{1}, x_{2}\right)}(x, y)+i V_{\left(x_{1}, x_{2}\right)}(x, y)\right] .
\end{gathered}
$$

Since

$$
U_{\left(x_{1}, 0\right)}(x, y)+U_{\left(0, x_{2}\right)}(x, y)=U_{\left(x_{1}, x_{2}\right)}(x, y)
$$

and

$$
V_{\left(x_{1}, 0\right)}(x, y)+V_{\left(0, x_{2}\right)}(x, y)=V_{\left(x_{1}, x_{2}\right)}(x, y) .
$$

The proof is now complete. We note that another way of writing the CauchyRiemann equations is

$$
F_{\left(x_{1}, 0\right)}(x, y)+i F_{\left(0 . x_{1}\right)}=0 .
$$

Definition 1 . Let $K$ be a large convex subset of $\bar{G}$. Define $H^{2}(K)$ to be

$\left\{F \mid F\right.$ analytic at each point of $\left.K \times \hat{G}, \sup _{x \in K} \int|F(x, y)|^{2} d y<\infty\right\}$

(the integral refers to the left-invariant Haar measure on $G$ ).

THEOREM 2. $H^{2}(K)$ is a Hilbert space with point-wise addition and inner product

$$
\left(F_{1}, F_{2}\right)=\sup _{x \in K} \int_{\vec{G}} F_{1}(x, y) \overline{F_{2}(x, y) d y} \quad(\|F\|=\sqrt{(F, F)})
$$


The proof will follow from a series of Lemmas.

LEMMA 2.1. If $\left\{F_{n}\right\}$ is a sequence in $H^{2}(K)$ such that $\left\|F_{n}-F_{m}\right\| \rightarrow 0$ as $n, m \rightarrow \infty$ then $F_{n}-F_{m}$ converges uniformly to zero on compact subsets of $K \times \hat{G}$.

Proof. Suppose $\left\|F_{n}-F_{m}\right\| \rightarrow 0$ and there exists a compact $K$ such that $F_{n}-F_{m} \rightarrow 0$ uniformly on $K$. Since $\left\|F_{n}-F_{m}\right\| \rightarrow 0,\left|F_{n}(x, y)-F_{m}(x, y)\right|$ is bounded on $K$ for each $n, m$. Let

$$
g_{n m}(K)=\sup _{(x, y) \in K}\left|F_{n}(x, y)-F_{m}(x, y)\right| .
$$

Since $K$ is compact, for each $n, m$ there exists $p_{n m}=\left(x_{n m}, y_{n m}\right)$ in $K$ such that

$$
g_{n m}(K)=\left|F_{n}\left(p_{n m}\right)-F_{m}\left(p_{n m}\right)\right| .
$$

Now since $F_{n}-F_{m} \rightarrow 0$ uniformly on $K$, there exist two unbounded sequences $\left\{n_{k}\right\},\left\{m_{k}\right\}$ such that $g_{n_{k} m_{k}}(K)>\varepsilon$ for all $k$. Let $K_{\varepsilon}$ be the closure of $\left\{p_{k}\right\}, p_{n_{k} m_{k}}=p_{k}$. By the continuity of $\left|F_{n}(x, y)-F_{m}(x, y)\right|$ for each $k$ there is a neighbourbood $N_{k}$ of $p_{k}$ such that $p \in N$ implies $\left|f_{n_{k}}(p)-f_{m_{k}}(p)\right|>\varepsilon / 2$.

$K_{\varepsilon}$ is covered by the collection of such neighbourhoods and as a closed subset of a compact set, $K_{\varepsilon}$ is compact hence there exists a finite subscover $N_{k_{1}}, \ldots, N_{k_{v}}$. Let

$$
M_{k i}=\left\{y \mid y \in \hat{G}, \exists \times \ni(x, y) \in N_{k_{i}}\right\} .
$$

Now we note that

$$
\left\|F_{n}-F_{m}\right\| \geqq \int_{\hat{q}}\left|F_{n}(x, y)-F_{m}\left(x_{k}, y\right)\right|^{2} d y
$$

for all $x_{k}$, and hence

$$
\left\|F_{n}-F_{m}\right\| \geqq \int_{M_{k_{i}}}\left|F_{n}\left(x_{k_{i}}, y\right)-F_{m}\left(x_{k_{i}}, y\right)\right|^{2} d y \geqq \frac{\varepsilon}{2} \mu\left(M_{k_{i}}\right)
$$

for $n=n_{k_{i}}, m=m_{k_{i}}$. If now $\delta=\min \left(\mu\left(M_{k_{i}}\right), \ldots, \mu\left(M_{k_{i}}\right)\right)$ then

$$
\left\|F_{n}-F_{m}\right\| \geqq \varepsilon \delta / 2 \quad \text { for } \quad n=n_{k}, \quad m=m_{k} \quad \text { all } \quad k \text {, }
$$

which contradicts $\left\|F_{n}-F_{m}\right\| \rightarrow 0$ and we conclude that $F_{n}-F_{m} \rightarrow 0$ uniformly on $K$.

LEMMA 2.2. Let $F$ be analytic at each point of $K \times \hat{G}, K$ a large convex subset of $\bar{G}$. If $K$ is a compact subset of $K \times \hat{G}$ then $F$ is uniformly analytic on $K$, i.e. given $\varepsilon>0,\left(x_{1}, x_{2}\right) \in \bar{G} \times \bar{G}$ there exists $\delta>0$ such that

$$
\frac{\mid F\left(x+u x_{1}, y x_{2}[u]\right)-F(x, y)}{u}-F_{\left(x_{1}, x_{2}\right)}(x, y)<\varepsilon
$$

for $0<|u|<\delta$ and all $(x, y) \in K$. 
Proof. Let $\varepsilon>0,\left(x_{1}, x_{2}\right) \in \bar{G} \times \bar{G}$, then for each $\left(x_{0}, y_{0}\right) \in K$ set $N\left(x_{0}, y_{0}\right)=\left\{(x, y)\left|\frac{F\left(x+u x_{1}, y x_{2}[u]\right)-F(x, y)}{u}-F_{\left(x_{1} x_{2}\right)}(x, y)\right|<\varepsilon\right.$ if $\left.0<|u|<\delta\right\}$

( $\delta$ is taken to be any non-zero value such that $0<|u|<\delta$ implies

$$
\left.\left|\frac{F\left(x_{0}+u x_{1}, y_{0} x_{2}[u]\right)-F\left(x_{0}, y_{0}\right)}{u}-F_{\left(x_{1}, x_{2}\right)}\left(x_{0}, y_{0}\right)\right|<\varepsilon\right) .
$$

Then $\bigcup_{\left(x_{0}, y_{0}\right) \in K} N\left(x_{0}, y_{0}\right)$ is an open covering of $K$, which is compact. Then there exist a finite number of points $\left(x_{1}, y_{1}\right),\left(x_{2}, y_{2}\right), \ldots,\left(x_{n}, y_{n}\right)$ associated $\delta$ 's $\delta_{1}, \ldots, \delta_{n}$ and neighbourhoods $N\left(x_{1}, y_{1}\right), \ldots, N\left(x_{n}, y_{n}\right)$ which is a finite subcovering of $K$. Let $\delta=\min \left(\delta_{1}, \ldots, \delta_{n}\right)$ and the result follows.

LEMMA 2.3. Let $F(x, y)$ be defined in a neighbourhood of $(x, y) \in \bar{G} \times \hat{G}$. Then $a$ necessary and sufficient condition that $F$ be analytic (in the sense of Definition 1) is that $g(z)$ be analytic at $z=0$ for all $\left(x_{1}, x_{2}\right) \in \bar{G} \times \bar{G}$ where $g_{\left(x_{1}, x_{2}\right)}(z)=F\left(x+u x_{1}-\right.$ $\left.-v x_{2}, y x_{2}[u] x_{1}[v]\right), z=u+i v$.

Proof. (a) Writing $\frac{\partial}{\partial z}=\frac{1}{2}\left(\frac{\partial}{\partial u}+i \frac{\partial}{\partial v}\right)$ as is customary then $g_{x_{\mathrm{r}}}(z)$ analytic at $z=0$ implies

However

$$
\left.\frac{\partial g}{\partial z}\right|_{z=0}=0
$$

and

$$
\left.\frac{\partial g}{\partial u}\right|_{z=0}=\operatorname{Lim}_{u \rightarrow 0} \frac{F\left(x+u x_{1}, y x_{2}[u]\right)-F(x, y)}{u}=F_{\left(x_{1}, x_{2}\right)}(x, y)
$$

$$
\left.\frac{\partial g}{\partial v}\right|_{z=0}=\operatorname{Lim}_{v \rightarrow 0} \frac{F\left(x-v x_{2}, y x_{1}[v]\right)-F(x, y)}{v}=F_{\left(-x_{2}, x_{1}\right)}(x, y) .
$$

Hence $\left.\frac{\partial g}{\partial z}\right|_{z=0}=0$ implies

or

$$
F_{\left(x_{1}, x_{2}\right)}(x, y)+i F_{\left(-x_{2}, x_{1}\right)}(x, y)=0
$$

$$
F_{\left(x_{1}, x_{2}\right)}(x, y)=-i F_{\left(-x_{2}, x_{1}\right)}(x, y)
$$

which together with the observations that $-i\left(-x_{2}, x_{1}\right)=\left(x_{1}, x_{2}\right)$ provides the complex homogeneity required. The existence of the derivative follows from the existence of the derivative of $g$. Since the preceding assertions are reversible the foosd of the theorem is complete.

LEMMA 2.4. Let $\left\{F_{n}(x, y)\right\}$ be a sequence of complex-valued functions analytic at each point of $K \times \hat{G}, K$ large convex in $\bar{G}$. If $\left\{F_{n}\right\}$ converges uniformly on compact subsets of $K \times \hat{G}$ then the limit function is analytic at each point of $K \times \hat{G}$. 
Proof. Let $\left(x_{0}, y_{0}\right) \in K \times \hat{G},\left(x_{1}, x_{2}\right) \in \bar{G} \times \bar{G}$ and $\delta>0$.

$$
\begin{gathered}
M_{\left(x, x_{2}\right)}(\delta)=\left\{(x, y) \mid x=x_{0}+u x_{1}-v x_{2}, y=y_{0} x_{2}[u] x_{1}[v],(x, y) \in M,\right. \\
|z| \leqq \delta,(x, y) \in K \times \hat{G}\},
\end{gathered}
$$

then $M_{\left(x_{1}, x_{2}\right)}(\delta)$ is compact and is nonempty for some $\delta \neq 0$. We see then that $\left\{F_{n}\right\}$ converging uniformly on $M_{\left(x_{1}, x_{2}\right)}(\delta)$ is equivalent, to $\left\{g_{n}\right\}$ converging uniformly on $|z| \leqq \delta$ where

$$
g_{n}(z)=F_{n}\left(x_{0}+u x_{1}-v x_{2}, y_{0} x_{2}[u] x_{1}[v]\right) .
$$

Now $g_{n}(z)$ is analytic for $|z| \leqq \delta$ if and only if $F_{n}$ is analytic in $M_{\left(x_{1}, x_{2}\right)}(\delta)$ with respect to $\left(x_{1}, x_{2}\right)$ by Lemma 2.3. Therefore $\left\{g_{n}\right\}$ has an analytic limit for $|z| \leqq \delta$ if and only if $\left\{F_{n}\right\}$ has an analytic limit at $\left(x_{0}, y_{0}\right)$. Since $\left(x_{0}, y_{0}\right)$ and $\left(x_{1}, x_{2}\right)$ were arbitrary, the proof is complete.

Proof of Theorem 2. By Lemma 2.1 a Cauchy sequence in $H^{2}(K)$ is a Cauchy sequence in the topology of uniform convergence on compact subsets of $K \times \hat{G}$. From Lemma 2.4 the limit under this latter topology is analytic. From the usual $L^{2}$ topology we have that the limit function must be in $H^{2}(K)$. The algebraic closure of $H^{2}(K)$ follows in the usual way.

THEOREM 3. If $F(x, y)$ is analytic at $\left(x_{0}, y_{0}\right) \in K \times \hat{G}$, for all $n, g\left(z_{1}, z_{2}, \ldots, z_{n}\right)$ is analytic at $\left(z_{1}, z_{2}, \ldots, z_{n}\right)=(0, \ldots, 0)$ where

$$
\begin{gathered}
g\left(z_{1}, \ldots, z_{n}\right)= \\
F\left(x_{0}+\sum_{i=1}^{n}\left(u_{i} x_{i}-v_{i} x_{i+n}\right), y_{0} \prod_{i=1}^{n} x_{i}\left[v_{i}\right] x_{i+n}\left[u_{i}\right]\right), \\
\left(x_{i}, x_{i+n}\right) \in \bar{G} \times \bar{G}, \quad i=1, \ldots n .
\end{gathered}
$$

Proof. By $n$ applications of Lemma 2.3, $g$ is analytic in $z_{i}$ for each $i$ and hence by the Osgood-Hartog Theorem [3] $g$ is analytic.

COROLLARY 3.1. If $F$ is analytic at $\left(x_{0}, y_{0}\right)$ then for all $\left(x_{1}, x_{2}\right) \in \bar{G} \times \bar{G}, F_{\left(x_{1}, x_{2}\right)}$ is analytic at $\left(x_{0}, y_{0}\right)$.

Proof. Let $n=2$ in Theorem 3 then $g\left(z_{1}, z_{2}\right)$ is analytic at $(0,0)$ and hence the double and iterated limits on the derivatives exist and are equal, i.e.

$$
\left.\frac{\partial^{2} g}{\partial \bar{z}_{1} \partial \bar{z}_{2}}\right|_{(0,0)}=\left.\frac{\partial^{2} g}{\partial \bar{z}_{2} \partial \bar{z}_{1}}\right|_{(0,0)}=0 \text {. }
$$

Evaluating these as in the proof of Lemma 2.3 we have

$$
\left(F_{\left(x_{1}, x_{3}\right)}\right)_{\left(x_{2}, x_{4}\right)}=\left(F_{\left(x_{2}, x_{4}\right)}\right)_{\left(x_{1}, x_{3}\right)}
$$

and both exist and are complex homogeneous functions of $\left(x_{1}, x_{3}\right),\left(x_{2}, x_{4}\right)$. By repeated applications of this corollary we have that $F$ analytic implies the existence of all higher orders of derivatives. 
If we re-define analyticity then we can obtain a weak form of Osgood-Hartog Theorem for these functions.

DeFINITION 4. Let $F(x, y)$ be defined in a neighbourhood of $\left(x_{0}, y_{0}\right) \in \bar{G} \times \hat{G}$. Then $F$ is said to be analytic in $x_{1}$ at $\left(x_{0}, y_{0}\right)$ if

$$
\operatorname{Lim}_{u+0} \frac{F\left(x_{0}+u x_{1}, y x_{1}[u]\right)-F(x, y)}{u}=F_{\left(x_{1}, x_{1}\right)}\left(x_{0}, y_{0}\right)
$$

exists and is a complex homogeneous function of $\left(x_{1}, x_{1}\right)$.

Theorem 5. (Weak Osgood-Hartog.) If $F$ is "analytic in $x_{1}$ at $\left(x_{0}, y_{0}\right)$ " for all $x_{1} \in \vec{G}$ then $F$ is analytic (in the sense of Definition 1).

PROOF. We note that

$$
\left(x_{1}, x_{2}\right)=\frac{1-i}{2}\left(x_{1}, x_{1}\right)+\frac{1+i}{2}\left(x_{2}, x_{2}\right)
$$

hence

$$
F_{\left(x_{1}, x_{2}\right)}=\frac{1-i}{2} F_{\left(x_{1}, x_{1}\right)}+\frac{1+i}{2} F_{\left(x_{2}, x_{2}\right)}
$$

and $F_{\left(x_{1}, x_{2}\right)}$ is a complex homogeneous function of $\left(x_{1}, x_{2}\right)$ since $F_{\left(x_{1}, x_{1}\right)}$ and $F_{\left(x_{2}, x\right)_{2}}$ are complex homogeneous functions of $\left(x_{1}, x_{1}\right),\left(x_{2}, x_{2}\right)$ respectively.

In conclusion we note two other results. Lemma 2.3 asserts that Definition 1 and Definition 4.10 [2] are equivalent. $H^{2}(K)$ is a subset of the set of $L^{2}$-analytic functions as defined by MACKEY [4] and hence by the Theorem, pp. 160 [4] each is the generalized Laplace Transform of a function in $L^{2}$ up to an equivalence relation.

(Received 2.5 June 1959)

UNIVERSITY OF ARIZONA

DEPT. OF MATH.

TUCSON, ARIZONA 85721

U.S.A.

\section{References}

[1] R. ARENS and I. M. SINGer, Generalized Analytic Functions, Trans AMS, 81 (1956).

[2] R. Eluot, Some results in Spectral Synthesis, Proc. Camb. Phil. Soc., 61 (1965), pp. 395.

[3] R. Gunning-R. Rossi, Analytic Functions of Several Complex Variables, Prentice-Hall, 1965.

[4] G. MACKEY, The Laplace Transform for Locally compact Abelian Groups, Proc. NAS, 34 (1948), pp. 156.

[5] D. E. Myers, Topologies for Laplace Transform Spaces, Pac. J. Math., 15 (3) (1965), pp. 957.

[6] D. E. Myers, The Algebra $H^{2}(K)$, Function Algebras, Scott-Foresman 1966, pp. 282.

[7] D. E. MYers, Some Subspaces of Analytic Functions, Duke J. Math., 35 (3) (1968), pp. 435. 